\title{
Congestion-Aware Downlink Scheduling for IEEE 802.16j Multi-Hop Relay Networks
}

\author{
Yi-Cheng Chan, Chang-Lung Wu, and Chin-Yu Lai \\ Department of Computer Science and Information Engineering \\ National Changhua University of Education \\ No. 2, Shi-Da Road, Changhua 500, Taiwan, R.O.C. \\ \{ycchan@cc, m95612015@mail, s94610022@mail\}.ncue.edu.tw
}

\begin{abstract}
In this paper, a congestion-aware downlink scheduling for IEEE 802.16j multi-hop relay networks is proposed. In the mechanism, a relay station (RS) can report its queue status of each downlink connection to the upstream station. Based on the reported information, the upstream station may properly schedule its downlink transmission and hence the system performance benefits. We focus on the following three issues to achieve the goal. First, to find a congested queue, we develop a measuring method to monitor queue statuses in a RS. Then, a report system is designed to carry the information. Finally, a congestion-aware downlink scheduling algorithm of a MR-BS and RS is devised to properly schedule the downlink transmission. According to the NS-2 simulation results, the proposed scheme effectively mitigates the congestion problem in a RS and improves the system performance. Compared with the contribution of downlink flow control presented in the IEEE 802.16j task group, the proposed scheduling scheme is able to increase the network throughput up to $60.11 \%$ and reduce the dropping ratio up to $26.21 \%$.
\end{abstract}

\section{Categories and Subject Descriptors}

C.2.1 [Computer-Communication Networks]: Network Architecture and Design-Wireless communication

\section{General Terms}

Algorithms

\section{Keywords}

IEEE 802.16j, Multi-Hop Relay Networks, Downlink Scheduling, Flow Control

\footnotetext{
* This work is sponsored in part by National Science Council, Taiwan, R.O.C., under grant NSC 97-2221-E-018-016.
}

Permission to make digital or hard copies of all or part of this work for personal or classroom use is granted without fee provided that copies are not made or distributed for profit or commercial advantage and that copies bear this notice and the full citation on the first page. To copy otherwise, to republish, to post on servers or to redistribute to lists, requires prior specific permission and/or a fee.

WICON'08, November 17-19, 2008, Maui, Hawaii, USA

Copyright 2008 ICST 978-963-9799-36-3.

\section{INTRODUCTION}

Wireless Metropolitan Area Networks (WMANs) are getting considerable attention in recent years because they can provide broadband wireless services over a large coverage area between network users and Internet Service Providers (ISPs). WMANs complement the existing last mile wired networks such as cable modem and xDSL. Due to the upcoming air interface technologies which promise to deliver high transmission data rates, WMANs become an attractive alternative. The Worldwide Interoperability for Microwave Access (WiMAX), based on the IEEE 802.16 standard, is presented as a technology that plays a key role in WMANs.

The IEEE 802.16 standard [1] specifies two basic operational modes: Point-to-MultiPoint (PMP) and Mesh (Optional). In the PMP mode, Subscriber Stations (SSs) or Mobile Stations (MSs) are organized into a cellular-like structure and connected to the Internet via the Base Station (BS). The BS controls the communications with multiple SSs in both downlink (DL) and uplink (UL) directions. By contrast, SSs can communicate directly with other stations in the Mesh mode.

However, due to significant loss of signal strength along the propagation path and the power constraint of MSs, the coverage area for a specific high data rate is often limited by the geographical size. Conventionally, this problem has been addressed by deploying BSs in a denser manner. However, this approach is less desirable for network operators because of high-cost BSs. Recently, a relay-based approach can be adopted to improve this problem. The IEEE 802.16j Multi-hop Relay (MR) task group attempts to amend the current IEEE 802.16e-2005 [2] standard and provides an attractive solution for the coverage extension and throughput enhancement $[9,10,11]$. The task group mainly introduces a low-cost a relay station (RS) to relay packets between a MR-BS and MSs. For providing backward compatibility, MSs existing in conventional IEEE 802.16 should be still available in IEEE 802.16j MR networks. Compared with the Mesh mode, IEEE 802.16j has been identified with a better feasibility and efficiency due to the similarities in the MAC and PHY layers.

In IEEE 802.16j multi-hop relay networks, a RS may adopt distributed scheduling algorithms to arrange transmission time for each downstream RS and MS. In some scenarios, a RS should buffer packets for its downstream RSs and MSs. For example, when a MS wants to handoff or goes into the power saving mode. However, a MR-BS cannot perceive the queue status of its downstream RS. Packets may be contin- 
uing sent from the MR-BS to a congested queue. It may result in a serious congestion in the RS.

In this paper, we investigate and devise a congestionaware downlink scheduling for IEEE $802.16 \mathrm{j}$ multi-hop relay networks. In the proposed mechanisms, a RS can monitor its queue status of each downlink connection and report the connection state to its upstream MR-BS or RS. Therefore, a measuring method is designed to calculate the average queue length of each downlink connection. Furthermore, a report scheme is developed to carry the measured information to the upstream stations. The upstream MR-BS or RS can properly schedule its downlink transmission based on the reported message. Compared with the downlink flow control [18] presented in the IEEE $802.16 \mathrm{j}$ task group, the simulation results show that the proposed mechanism is able to increase the network throughput up to $60.11 \%$ and decrease the dropping ratio up to $26.21 \%$.

The rest of this paper is organized as follows. In Section 2 , we introduce the background knowledge of IEEE 802.16e2005 and IEEE 802.16j. The congestion-aware downlink scheduling algorithm is proposed to solve the congestion problem in Section 3. In Section 4, we show the simulation results to discuss the performance of our mechanism. To demonstrate the efficiency, we compare the proposed scheme with the downlink flow control [18]. Finally, the conclusions and future work are given in Section 5.

\section{RELATED WORK}

This section provides an overview of the IEEE 802.162005e and IEEE 802.16j specifications relevant to the scheduling and data transmission at the MAC layer. In addition, we describe the downlink flow control that is proposed in the IEEE 802.16j task group. For further details related to the IEEE 802.16 standard, please refer to [2, 3, 14].

\subsection{IEEE 802.16e-2005}

Two primary channel access mechanisms are defined in the IEEE 802.16 specification: Orthogonal Frequency Division Modulation (OFDM) and Orthogonal Frequency Division Multiplexing Access (OFDMA). We mainly focus on the OFDMA air interface in this paper. In the PMP mode, downlink (from BS to SS) and uplink (from SS to BS) data transmissions occur in separate subframes. In the downlink subframe, a BS transmits a burst of MAC Protocol Data Units (PDUs). Since the transmission is broadcasted, all SSs listen to the data transmitted by the BS. A SS only requires to process PDUs that are addressed to itself. On the other hand, a SS transmits a burst of MAC PDUs to the BS in a Time-Division Multiple Access (TDMA) manner in the uplink subframe. Downlnk and uplink subframes are duplexed using Frequency-Division Duplex (FDD) or TimeDivision Duplex (TDD).

The MAC protocol is connection-oriented. All connections for both data transport and management are unidirectional. A downlink and an uplink subframes are concatenated in a MAC frame, as shown in Figure 1. A MAC frame is a unit of time used for representing the transmission status between a BS and SSs. Each frame consists of a preamble, MAPs, a number of downlink and uplink bursts, transition gaps and a ranging region. The preamble helps SSs perform synchronization and channel estimation. The DL-MAP and UL-MAP messages notify SSs of the bursts allocated to them in the downlink and uplink direction, re- spectively. The transition gaps (i.e. TTG and RTG) give the stations sufficient time to switch from the transmission mode to reception mode, or vice versa. The ranging region is provided for new SSs joining the network. At the beginning of each frame, the BS schedules the downlink and uplink transmissions according to the connection queues and bandwidth requests, respectively. The scheduled results are included in the DL-MAP and UL-MAP messages and broadcasted by the BS. Each SS gains the transmission interval by decoding the messages.

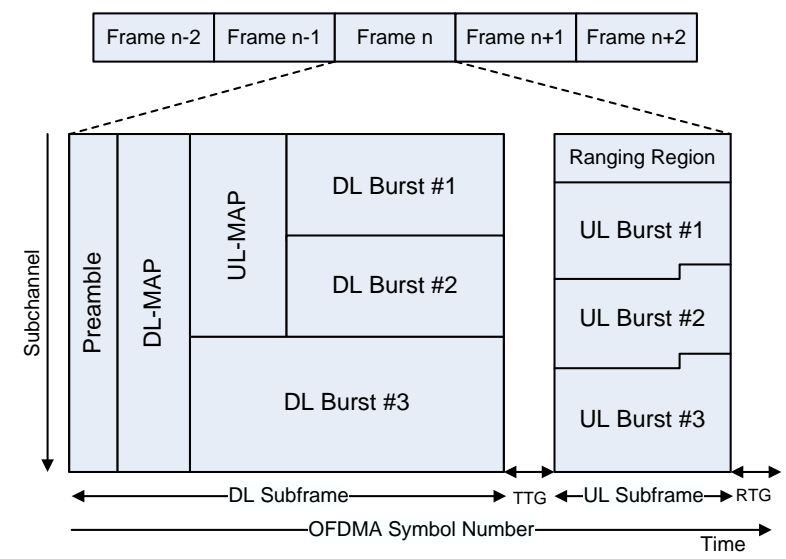

Figure 1: An OFDMA frame in TDD mode.

Five scheduling services are defined in the IEEE 802.162005e specifications in order to meet the QoS requirements of varied applications. Those are Unsolicited Grant Service (UGS), Real-Time Variable-Rate (RT-VR), Extended RealTime Variable-Rate (ERT-VR), Non-Real-Time VariableRate (NRT-VR), and Best Effort (BE). The scheduling services represent the data handling mechanisms supported by the MAC scheduler. Each connection is associated with a single scheduling service. A scheduling service is determined by a set of QoS parameters. These parameters are managed using the exchanges of MAC management messages.

\subsection{IEEE 802.16j}

The sample topology of IEEE $802.16 \mathrm{j}$ multi-hop relay network is shown in Figure 2. There are two kind of wireless links specified in the draft: access link and relay link. A BS that is capable of supporting multi-hop relay is called MR-BS. The access link is either a downlink or an uplink direction between a MR-BS and a MS defined in the IEEE 802.16-2004 specification. The relay link is used for relaying data between a MR-BS and a MS or between a pair of RSs.

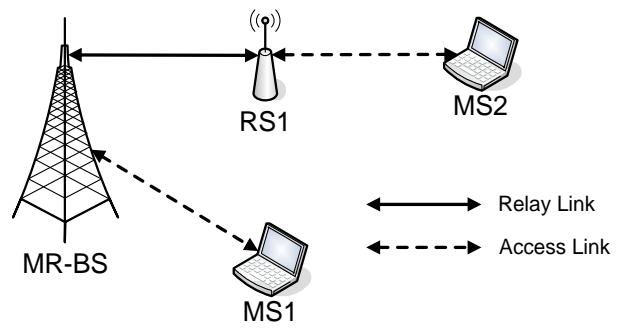

Figure 2: The multi-hop network topology. 
The OFDMA is the channel access mechanism of PHY layer in the IEEE 802.16j draft [3]. A new frame structure as shown in Figure 3 is presented, where each downlink subframe or uplink subframe is divided into access zone and relay zone. The access zone and relay zone are used for access links and relay links, respectively. Each DL/UL subframe may have more than one relay zone. There are two types of RSs have been defined: non-transparent RS and transparent RS. The non-transparent RS works as a BS at the access zone, hence a MS can recognizes the RS. This type of RS is allowed to transmit its own preamble, MAPs and other management messages to MSs over the access zone. The non-transparent RS acting as a MS switches to the receiver mode at the DL relay zone and the transmitter mode at the UL relay zone. For the transparent RS, it does not have its own preamble and MAPs. It looks transparent to MSs and only relays data for the MR-BS and MSs.

There are two scheduling modes described in the latest IEEE 802.16j draft: centralized scheduling and distributed scheduling. The centralized scheduling is that a MR-BS determines the bandwidth allocations and generates the corresponding MAPs for all access and relay links in the MR-cell. For the distributed scheduling, the MR-BS and each RS in the MR-cell determine the bandwidth allocations and generate the corresponding MAPs for the access and relay links of downstream stations. The non-transparent RS can transmit its own preamble and other management messages so that it can adopt the both scheduling schemes. The transparent RS only uses the centralized scheduling because it cannot produce the control messages.

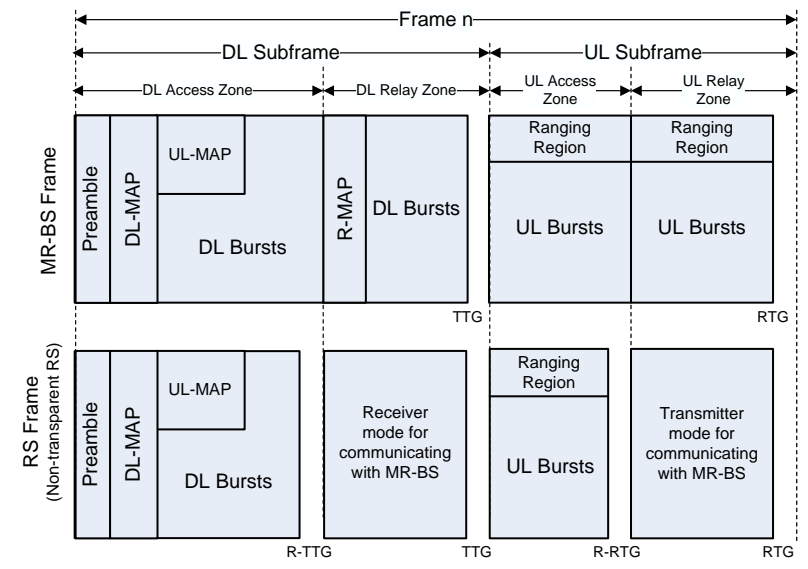

Figure 3: The frame structure for IEEE 802.16j multi-hop relay networks.

\subsection{Downlink Flow Control}

When the IEEE 802.16j multi-hop relay network operates using the distributed scheduling mode, a RS receives downlink data from the MR-BS. However, the MR-BS schedules the downlink transmission without regarding for wireless links beyond its control. A RS may accumulate downlink packets destined for its downstream stations because of temporary reductions in the capacity of its access or relay links. In this situation the RS needs to indicate to the MR-BS that it is temporarily not able to accept downlink traffic. As the buffered packets have been cleared, the RS then notifies the MR-BS that it is able to accept more packets.
A downlink flow control contribution [18] has been proposed in the IEEE $802.16 \mathrm{j}$ task group to mitigate the above issue. To prevent the congestion occurs in a RS, a new header is defined to allow RSs informing the upstream RS or MR-BS of its credit. In this contribution, the credit represents the state of the flow control protocol and number of bytes of downlink traffic that the upstream RS or MR-BS can send to the downstream RS. Nevertheless, it does not provide per queue information of each downlink connection. A MR-BS may still transmit packets to a congested connection as usual. It will result in a severe packet dropping situation. Accordingly, a more detailed study to resolve the flow control issue in IEEE $802.16 \mathrm{j}$ multi-hop relay networks is needed.

\section{PROPOSED SCHEME}

In this section, based on the considerations described in Subsection 2.3, we propose a congestion-aware downlink scheduling that consists of the following three mechanisms:

1. A queue status measurement method that is needed by a RS to contiguously monitor its each downlink queue in order to find out the congested connection.

2. A report mechanism is developed for notifying the upstream MR-BS or RS of the congested connection.

3. A downlink scheduling algorithm that is designed to schedule the downlink connections appropriately based the received message.

\subsection{Queue Status Measurement}

To detect the congested connection, the RS needs a method to obtain the status of each downlink connection. We modify the Random Early Detection (RED) [8] algorithm to estimate each downlink connection. The adjusted pseudo code is presented in Figure 4.

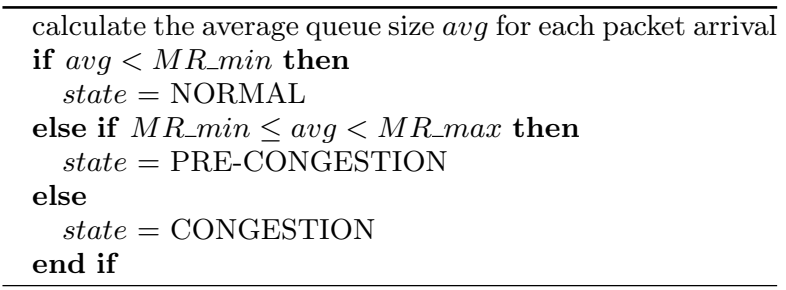

\section{Figure 4: Queue status measurement.}

For each packet arrival, the RS classifies the packet to a downlink queue according to its connection ID. Then, an Exponentially Weighted Moving Average (EWMA) scheme is adopted to calculate the average length for the downlink queue. Thus, the temporal increase of the queue length that results from bursty traffic or transient congestion can be eliminated. The EWMA equation is shown below:

$$
a v g \leftarrow\left(1-w_{q}\right) \cdot a v g+w_{q} \cdot q,
$$

where avg is the average length of the downlink queue, $w_{q}$ represents the weighted factor ranging between 0 and 1 , and $q$ stands for the current queue length.

We have to modify the RED algorithm so that it can operate normally in the IEEE $802.16 \mathrm{j}$ multi-hop relay networks. 
In the first place, $w_{q}$ is an important factor in our proposed mechanism. If $w_{q}$ is too large, the transient congestion in RSs cannot be eliminated by the EWMA equation. Nevertheless, if $w_{q}$ is set too low, the EWMA equation is unable to detect the initial congestion in RSs. In this study, we set the value of $w_{q}$ to 0.002 that is the same as the setting of RED algorithm [8]. Then, the values of $M R \_$min and $M R \_$max need to be decided. By the equation (1) and the value of $w_{q}$ is set to 0.002 , if the queue length is continually increased from 0 to 50 packets, the average queue size $(\mathrm{avg})$ is raised from 0 to 3 packets approximately. Assume that the RS's queue size is set to 50 packets. We can set the $M R \_$min to 3 packets and the $M R \_$max to triple of $M R \_$min. Since it expresses that the packets are accumulated and the queue may be overflow. According to the above description, the $\mathrm{RS}$ can assign the downlink connection to the following three states:

- NORMAL: it represents that the queue is not congested.

- PRE-CONGESTION: the queue may be congested soon.

- CONGESTION: it depicts the queue in congestion state.

\subsection{Report Mechanism}

After the RS obtains the status of each downlink connection, it must notify the upstream MR-BS or RS of the information. The upstream station can schedule the downlink connections based on the reported message. Hence, the reported mechanism needs to be developed. In the previous subsection, we have specified the three states for each connection. Therefore, the report message needs to contain two bits to represent the three states. Furthermore, the RS has a responsibility to inform the upstream MR-BS or RS of the congested Connection ID (CID). To sum up, the reported information should consist of the measured status (2 bits) and the connection ID (16 bits) at least.

In the IEEE 802.16 specifications, the Extended Subheader Field (ESF) bit in the MAC header is defined to indicate the extended subheader group is presented, and is applicable in the both DL or UL direction. If the ESF bit is set to 1, the extended subheader group will follow the MAC header immediately. The extended subheader group is composed of extended subheader group length ( 8 bits), extended subheader type ( 7 bits) and extended subheader body (variable). By using the ESF, we can create a congestion-aware extended subheader as shown in Table 1 to inform the upstream stations of the connection state and the connection ID.

Table 1: Congestion-aware extended subheader

\begin{tabular}{|c|c|l|}
\hline Name & Length & Description \\
\hline \hline & & 0b00: NORMAL \\
Connection state & 2 bits & 0b01: PRE-CONGESTION \\
& & 0b10: CONGESTION \\
& & 0b11: Reserved \\
\hline CID & 16 bits & Connection ID \\
\hline Reserved & 6 bits & Reserved \\
\hline \multicolumn{2}{|l}{} \\
\hline
\end{tabular}

The state transition diagram for each downlink connection is shown in Figure 5. When the connection state changes, the RS should notify its upstream station of the connection state. Because the RS also needs to relay the uplink packets to its upstream station, it can embed the congestion-aware extended subheader in the packet of the current uplink connection. The RS can inform the upstream station of the congestion information through this report manner. On the other hand, the EWMA scheme is adopted to calculate the average queue length of each downlink connection. The main advantage of EWMA is to eliminate bursty. For that reason, the connection state may not be changed violently.

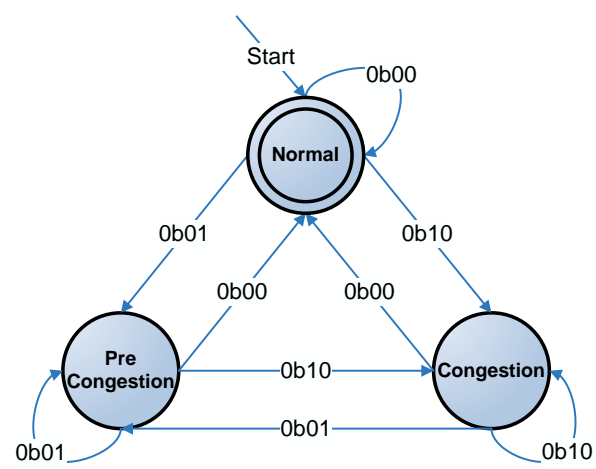

Figure 5: The state transition diagram of a connection.

\subsection{Congestion-Aware Downlink Scheduling}

The IEEE 802.16 standards left the scheduling algorithms that determine the DL and UL bandwidth allocation undefined. Thus, there are many researches $[4,6,7,13,15,16$, 19] that propose the scheduling methods and performance evaluation to solve the undefined part. In this study, we introduce a congestion-aware downlink scheduling in the upstream stations for IEEE $802.16 \mathrm{j}$ multi-hop relay networks. The proposed scheduling scheme can arrange the transmission time for downlink connections based on the reported message to mitigate the congestion in RSs. Three principles are considered in designing the scheduling algorithm for a RS: low complexity, congestion-aware, and fairness.

Deficit Round Robin (DRR) [17] is adopted as the scheduling fundamental in the MR-BS or RS. DRR is a low-complexity and fairness scheduling algorithm. It mainly uses two important parameters: Quantum (Q) is the number of bytes allocated to a connection within the period of one round. Deficit Counter (DC) stands for the remainder number of bytes provided for next round. Each queue $i$ maintains a $D C_{i}$. When DRR serves a queue $i$, it adds $\mathrm{Q}$ to $D C_{i}$. If the packet size in the head of queue $i$ is less than $D C_{i}$, the packet can be sent, and $D C_{i}$ is subtracted from the packet size. Otherwise, DRR then serves the next queue $i+1$.

We reform the DRR algorithm to allow it can be aware of the connection states of downstream RSs. The scheduling algorithm in the MR-BS or RS should stop the downlink transmission of a congested connection according to the congestion-aware extended subheader. The pseudo code of the congestion-aware downlink scheduling is shown in Figure 6 . The proposed algorithm is designed according to the following bases:

1. Each MR-BS or RS maintains the state transition of connections. When the upstream station receives the 
reported message, it schedules the downlink connections based on the connection state.

2. A Pause Counter (PC) is added to each queue. When the scheduler serves a queue $i$, it will not to schedule the packets in queue $i$ and subtract $P C_{i}$ from one if $P C_{i}$ is greater than zero.

3. A procedure is designed to update the pause counter of each downlink connection based on the connection state.

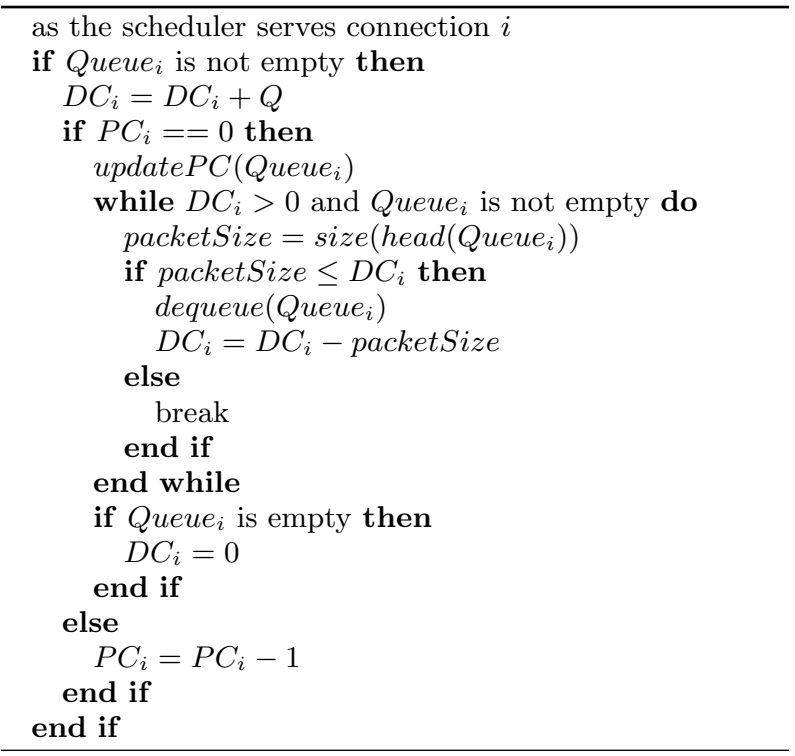

Figure 6: The proposed scheduling scheme.

We specify the operation of proposed scheduling scheme by using the example shown in Figure 7. Firstly, in Figure $7(\mathrm{a})$, the scheduler adds Q (500) to DC (0) of CID1. Since the DC (500) is greater than the packet size (200) in the head of CID1 queue, this packet should be scheduled to transmit. However, the pause counter of CID1 is equal to one, the proposed scheduling scheme subtracts the value of $\mathrm{PC}$ from one and skips the connection CID1. In Figure 7(b), the PC of CID2 is equal to zero and the value of DC is not less than the packet size (500) so that the packet is scheduled to transmit.

The pause counter of each downlink connection is updated by the upstream station according to the following principles: if the state is NORMAL, the upstream station sets the pause counter to zero. It represents that the bandwidth is not controlled, and the scheduler serves the connection normally. The pause counter is set to one if the connection is in the PRE-CONGESTION state. It means that the bandwidth allocation for this connection is cut by half in the state. If the state is CONGESTION, the upstream station sets the pause counter to 25 . In the next subsection, we will discuss the value of pause counter in different situations.

Based on the above description, the proposed congestionaware downlink scheduling has the following benefits:

- Low complexity: The proposed scheduling scheme inherits the low complexity of DRR algorithm. Consequently, it is easy to be implemented in the MR-BS or RS.

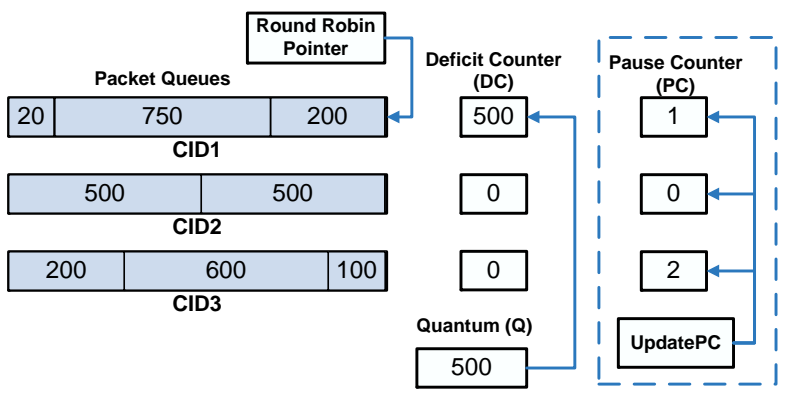

(a) The scheduler serves CID1

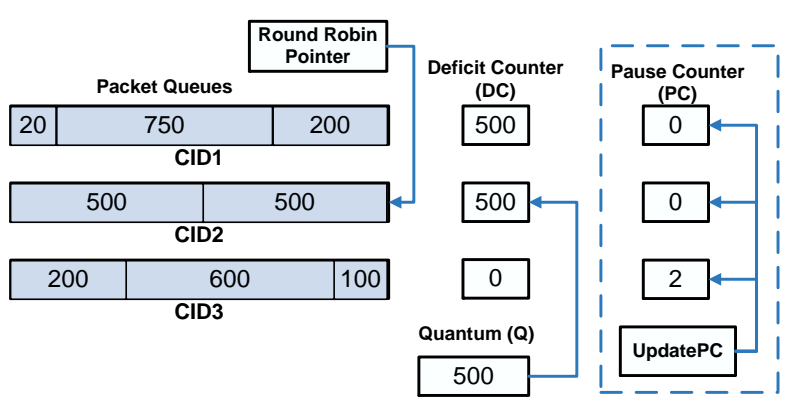

(b) The scheduler serves CID2

Figure 7: An example of the proposed scheduling.

- Congestion aware: The downstream RS can report the state of the congested connection. The upstream station schedules the downlink connections according to the reported information. The proposed pause counter is able to stop the transmission of congested connection temporarily in order to mitigate the congestion problem of the downstream RS.

- Fairness: The proposed mechanism keeps the fairness of each connection depends on the deficit counter and the quantum.

\subsection{Discussion of Pause Counter}

In this subsection, we discuss the value range of pause counter in the different QoS requirements. There are five scheduling services defined in the IEEE 802.16 specifications. Each scheduling service is associated with a set of QoS parameters including minimum reserved traffic rate, maximum sustained traffic rate, tolerated jitter or maximum latency. Therefore, some principles of the pause counter values should be discussed according to the QoS parameters.

The bandwidth for connection $i$ should be allocated by a upstream station as follows:

$$
B_{\min } \leq B_{\text {allocated }} \leq B_{\max },
$$

where $B_{\min }$ is the minimum reserved traffic rate, $B_{\text {allocated }}$ represents that the allocated bandwidth, and $B_{\max }$ is the maximum sustained traffic rate. We assume that $B_{\text {allocated }}^{\prime}$ is the bandwidth allocation when the value of pause counter is greater than zero. Therefore, according to the above considerations, the following inequality should be satisfied:

$$
\left(B_{\text {allocated }}^{\prime}=\frac{B_{\text {allocated }}}{P C_{i}+1}\right) \geq B_{\text {min }},
$$

The bandwidth allocation controlled by the pause counter is greater than or equal to the minimum reserved traffic rate. 
Accordingly, the value range of pause counter for connection $i$ is given as follows:

$$
0 \leq P C_{i} \leq \frac{B_{\text {allocated }}}{B_{\min }}-1
$$

Some scheduling services have the requirements of maximum latency or tolerated jitter, such as UGS, RT-VR and ERT-VR. Hence, we need to take the two restrictions into account when calculating the value range of pause counter. The latency is the time interval between the packet received in the transmission station and the reception of the packet in the peer station. The pause counter directly affects the queuing delay. Therefore, the following inequality needs to be satisfied:

$$
P C_{i} \cdot F D+T_{\text {delay }} \leq L_{\max },
$$

where $F D$ is the frame duration, $T_{\text {delay }}$ is the communication delay consisting of processing delay, queuing delay, transmission delay and propagation delay, and $L_{\max }$ represents the maximum latency. The tolerated jitter represents the maximum delay variation for this connection. Assume that $T_{\text {delay }}$ of each packet is equal. Accordingly, based on the maximum latency, the pause counter needs to satisfy the following inequality:

$$
\left|\left(P C_{i, t}-P C_{i, t-1}\right) \cdot F D\right| \leq J_{\text {tolerated }},
$$

where $P C_{i, t}$ is the value of pause counter at the $t$ interval, and $J_{\text {tolerated }}$ is the tolerated jitter.

\section{PERFORMANCE EVALUATION}

In this section, we present the performance evaluation in detail. First, the simulation environment including the parameter setting of MAC and PHY layers is described. Then, we evaluate the performance of the proposed mechanism and compare with that of the downlink flow control in different simulation scenarios.

\subsection{Simulation Environment}

In order to evaluate the proposed mechanism, we have modified the NIST WiMAX module [5] in the NS-2 simulator. The modified module supports the simulation of the IEEE $802.16 \mathrm{j}$ multi-hop relay networks. The MAC layer includes the main functions of the IEEE $802.16 \mathrm{j}$ draft, such as downlink and uplink transmissions for access links or relay links, management and transport connections, and ranging periods. The PHY implementation is OFDM. The size of OFDM symbol and the bandwidth support for different modulation and channel coding schemes are specified in Table 2. The system parameters in the NS-2 simulator are listed in Table 3. The MR-BS and RS allocate the frame structures for the TDD mode. The packing and fragmentation in the MR-BS or RS are disabled in all simulation scenarios. Automatic Repeat reQuest (ARQ) is not implemented in our simulation. The UDP protocol is adopted at the transport layer, and the packet size is set to 128 bytes. The sending rate of CBR traffic is set to $500 \mathrm{~Kb} / \mathrm{s}$. The VBR source is a Pareto distribution ON-OFF source with shape parameter 1.5. During ON periods, the VBR source sends data at $1 \mathrm{Mb} / \mathrm{s}$. The average sending rate of $\mathrm{VBR}$ source is set to $500 \mathrm{~Kb} / \mathrm{s}$.

\subsection{Simulation Scenario 1}

Table 2: Symbol size for the OFDM PHY

\begin{tabular}{|c|c|c|}
\hline Modulation \& Coding & $\begin{array}{c}\text { Symbol size } \\
\text { (bits) }\end{array}$ & $\begin{array}{c}\text { Bandwidth } \\
\text { (Kbps) }\end{array}$ \\
\hline \hline BPSK-1/2 & 88 & 1573 \\
\hline QPSK-1/2 & 184 & 3289 \\
\hline QPSK-3/4 & 280 & 5005 \\
\hline 16QAM-1/2 & 376 & 6721 \\
\hline 16QAM-3/4 & 578 & 10332 \\
\hline 64QAM-2/3 & 760 & 13585 \\
\hline 64QAM-3/4 & 856 & 15301 \\
\hline
\end{tabular}

Table 3: Parameters setting in the NS-2

\begin{tabular}{|l|l|}
\hline Parameter & Value \\
\hline \hline PHY & OFDM \\
\hline Frequency bandwidth & $5 \mathrm{MHz}$ \\
\hline Frame duration & $8 \mathrm{~ms}$ \\
\hline Frames per second & 125 \\
\hline Symbols per frame & 143 \\
\hline Connection queue size (BS) & 100 packets \\
\hline Connection queue size (RS) & 50 packets \\
\hline Duplex mode & TDD \\
\hline Packing/fragmentation & OFF \\
\hline DL ratio & 0.5 \\
\hline DL access ratio & 0.5 \\
\hline UL access ratio & 0.5 \\
\hline
\end{tabular}

The network topology used in the first simulation scenario is depicted in Figure 8. The network includes one MR-BS, one RS, two hosts and two MSs. Both the MS1 and MS2 are connected to the MR-BS via the RS. The two hosts are attached to the MR-BS using wired links. Each MS establishes a pair of data connections for downlink and uplink to the MR-BS through the RS. When the simulation starts, the Host1 and Host2 transmit the CBR or VBR traffic using the UDP protocol to the MS1 and MS2, respectively. In this scenario, the MS1 communicates with the RS using different modulation and coding schemes listed in Table 4. For instance, the MS1 transmits or receives data using the QPSK-1/2 mode from 20 to 30 seconds. It should be noticed that the MS2 always uses the 64QAM-3/4 mode in this scenario. The purpose of Table 4 is to make the congestion happened in the $\mathrm{RS}$ so that we can estimate the performance of the congestion-aware downlink scheduling (CA) and the downlink flow control (DLFC).

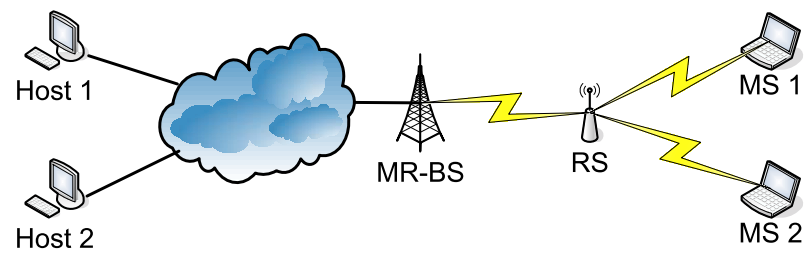

Figure 8: Network topology for scenario 1.

The simulation results of throughput are illustrated in Figure 9 and 10, and the information is gathered at the MS1 and MS2 for the two schemes. The dropping ratios of CBR and VBR traffics are shown in Figure 11 and 12, respectively. It is worth to mention that the dropping ratio represents the 
Table 4: Modulation and coding scenario.

\begin{tabular}{|c|c|}
\hline Simulation time (s) & Modulation and coding \\
\hline \hline $10-20$ & $64 \mathrm{QAM}-3 / 4$ \\
\hline $20-30$ & QPSK-1/2 \\
\hline $30-40$ & BPSK-1/2 \\
\hline $40-50$ & QPSK-1/2 \\
\hline $50-60$ & $64 \mathrm{QAM}-3 / 4$ \\
\hline
\end{tabular}

number of dropped packets to the number of received packets. The time interval from 0 to 10 seconds is left for the RS and MSs to join the network. From 10 to 20 seconds, the bandwidth is sufficient to support the data transmission, and no packet is dropped. With the modulation and coding scheme changes, the packets begin to be accumulated and dropped in the RS. The proposed mechanism can stop the transmissions of congested connections, and the saved bandwidth can be allocated to other connections. However, the mechanism of downlink flow control cannot perceive the congested connection, and the upstream MR-BS or RS may transmit the packets to the congested queue. The improvements of throughput and dropping ratio for CBR and VBR traffic are listed in Table 5. Overall, comparing to the downlink flow control, the proposed mechanism can increase the throughput up to $60.11 \%$ and decrease the dropping ratio up to $14.21 \%$ for the CBR traffic. For the VBR traffic, the throughput can be increased up to $51.18 \%$ and the dropping ratio can be decreased up to $10.09 \%$.

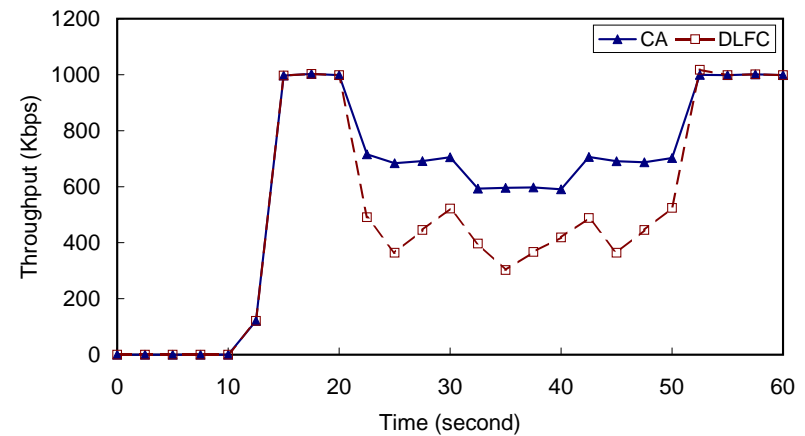

Figure 9: Throughput for scenario 1 (CBR).

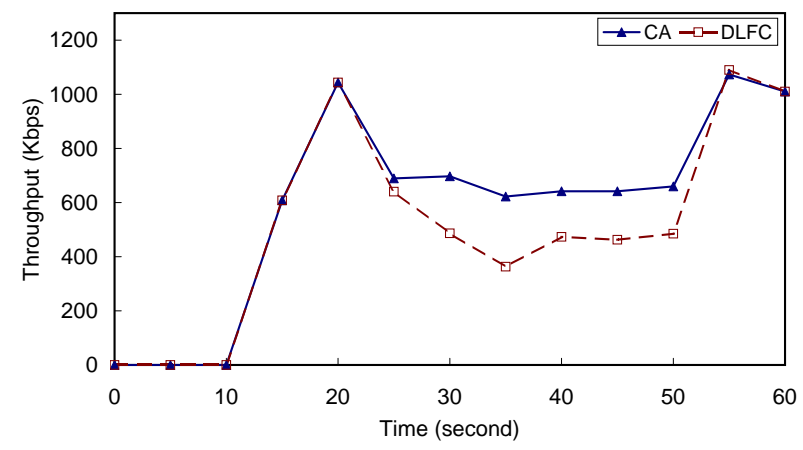

Figure 10: Throughput for scenario 1 (VBR).

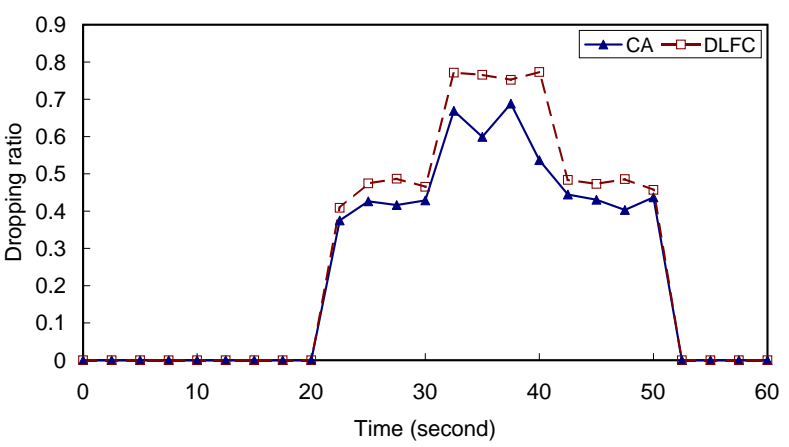

Figure 11: Dropping ratio for scenario 1 (CBR).

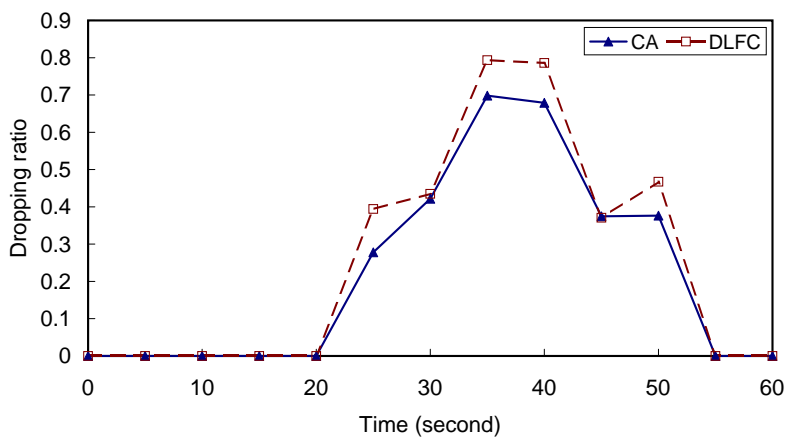

Figure 12: Dropping ratio for scenario 1 (VBR).

In this simulation scenario, the network topology is shown in Figure 13. The network consists of one MR-BS, two RSs, four hosts and four MSs. Each MS joins the network through their upstream RSs. The four hosts are connected to the MR-BS using wired lines. As the simulation starts, all hosts transmit the CBR or VBR traffic to the MSs using the UDP protocol, respectively. In this scenario, the MS1 and MS3 communicate with their upstream RSs using the scenario that described in Table 4 . The MS2 and MS4 always adopt the 64QAM-3/4 mode. We implement the proposed mechanism in the RS1, and the RS2 adopts the downlink flow control scheme. The purpose of this scenario is to estimate the system performance when both schemes operate in the same network.

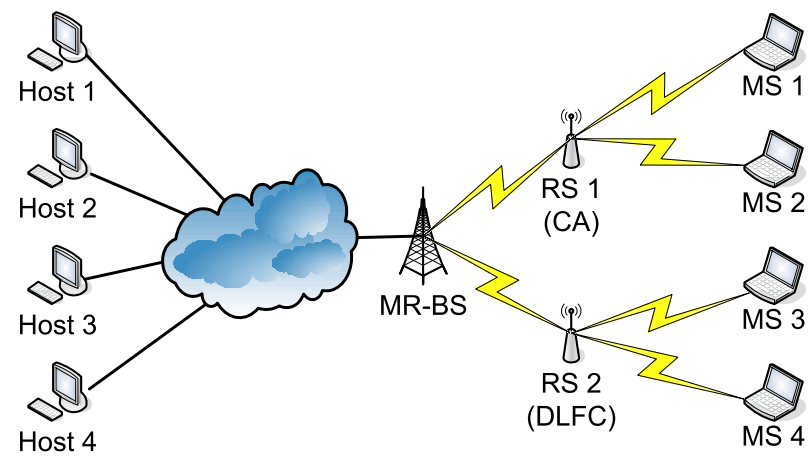

Figure 13: Network topology for scenario 2.

The throughputs of CBR and VBR traffics are depicted 
Table 5: The improvements for scenario 1.

\begin{tabular}{|c|c|c|}
\hline \multicolumn{3}{|c|}{ Network throughput } \\
\hline Time (s) & CBR & VBR \\
\hline $20-30$ & $53.52 \%$ & $23.06 \%$ \\
\hline $30-40$ & $60.11 \%$ & $51.18 \%$ \\
\hline $40-50$ & $52.97 \%$ & $37.34 \%$ \\
\hline \hline \multicolumn{3}{|c|}{ Dropping ratio } \\
\hline Time (s) & CBR & VBR \\
\hline $20-30$ & $4.72 \%$ & $6.46 \%$ \\
\hline $30-40$ & $14.21 \%$ & $10.09 \%$ \\
\hline $40-50$ & $4.60 \%$ & $4.36 \%$ \\
\hline
\end{tabular}

in Figure 14 and 15, and the simulation results of dropping ratio are shown in Figure 16 and 17. From 10 to 20 seconds, the bandwidth is enough to support the data transmission, and no packet is discarded. With the modulation and coding scheme changes, the packets begin to be dropped that results from the temporary reduction in the capacity of access links. As the two schemes operate in the same network, our proposed mechanism outperforms the downlink flow control in terms of throughput and dropping ratio. The improvements of throughput and dropping ratio are listed in Table 6. Overall, by using the proposed scheduling scheme, the throughput can be increased up to $46.49 \%$ and the dropping ratio can be decreased up to $26.21 \%$ for the CBR traffic. For the VBR traffic, the proposed mechanism can increase the throughput up to $59.34 \%$ and decrease the dropping ratio up to $14.74 \%$.

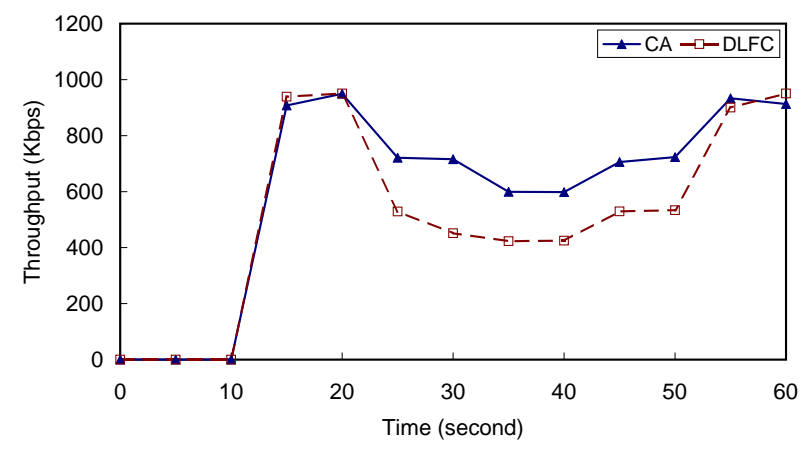

Figure 14: Throughput for scenario 2 (CBR).

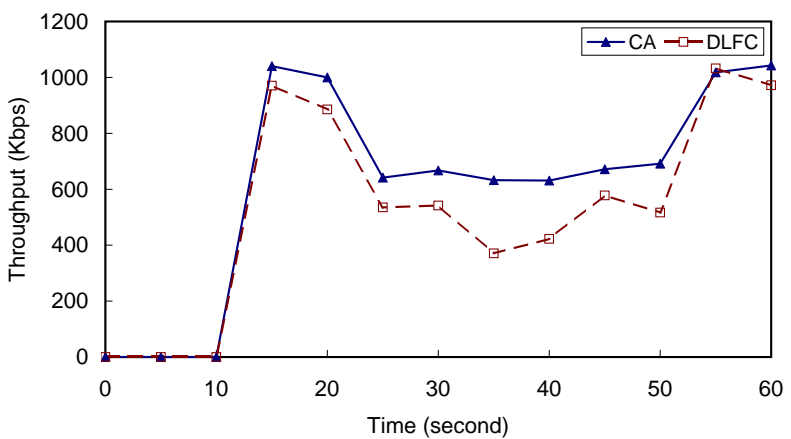

Figure 15: Throughput for scenario 2 (VBR).

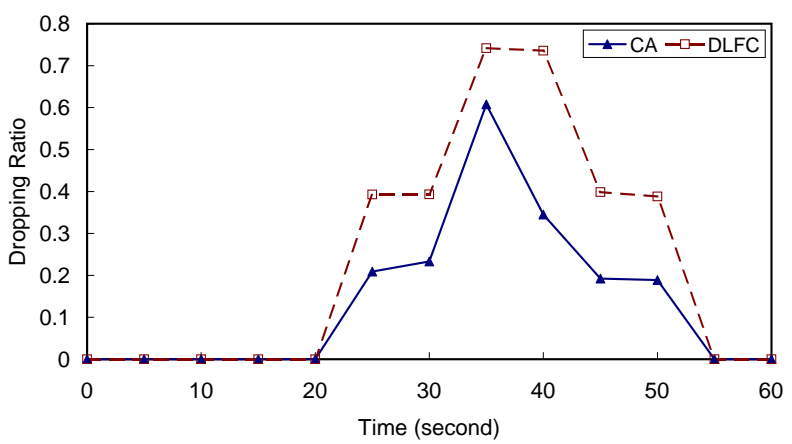

Figure 16: Dropping ratio for scenario 2 (CBR).

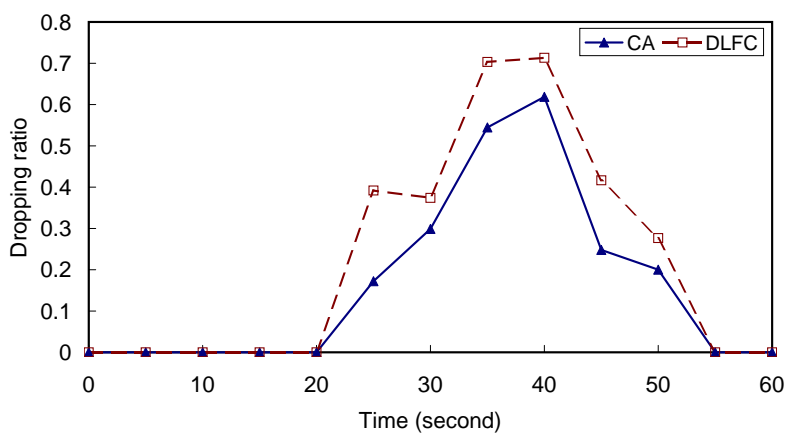

Figure 17: Dropping ratio for scenario 2 (VBR).

\subsection{Simulation Scenario 3}

We present the fairness feature of the proposed scheduling scheme in this simulation scenario. The network topology is similar to Figure 8. It consists of one MR-BS, one RS and a number of MSs and hosts. Each MS is connected to the MRBS through the RS. These hosts are connected to the MR-BS using the wire links, and transmit the VBR traffics to the MSs using the UDP protocol. In this simulation, the average sending rate of VBR source is set to $30 \mathrm{~Kb} / \mathrm{s}$. During ON period, the VBR source sends data at $60 \mathrm{~Kb} / \mathrm{s}$. It should be noticed that the RS and MSs use the 64QAM-3/4 mode to communicate with the MR-BS and RS, respectively. In order to verify the fairness feature of the proposed scheduling scheme, we adopt the widely used Jain's fairness index [12] given by:

$$
F=\frac{\left[\sum_{i=1}^{N} X_{i}\right]^{2}}{N \cdot \sum_{i=1}^{N} X_{i}^{2}},
$$

where $N$ is the number of the considered MSs and $X_{i}$ is the average throughput of the i-th MS. When the throughputs of all stations are the same, the fairness index is equal to 1 .

From the simulation results shown in Table 7 , we can find that the proposed scheduling scheme is able to preserve the fairness when the number of MSs is increased. This is because our proposed scheme inherits the good characteristics of DRR algorithm and keeps the fairness of each downstream station.

\section{CONCLUSIONS}

We propose a congestion-aware downlink scheduling for IEEE $802.16 \mathrm{j}$ networks in this paper. In the mechanism, 
Table 6: The improvements for scenario 2.

\begin{tabular}{|c|c|c|}
\hline \multicolumn{3}{|c|}{ Network throughput } \\
\hline Time (s) & CBR & VBR \\
\hline $20-30$ & $46.49 \%$ & $21.48 \%$ \\
\hline $30-40$ & $41.25 \%$ & $59.34 \%$ \\
\hline $40-50$ & $34.44 \%$ & $10.37 \%$ \\
\hline \hline \multicolumn{3}{|c|}{ Dropping ratio } \\
\hline Time (s) & CBR & VBR \\
\hline $20-30$ & $17.19 \%$ & $14.74 \%$ \\
\hline $30-40$ & $26.21 \%$ & $12.66 \%$ \\
\hline $40-50$ & $20.28 \%$ & $12.26 \%$ \\
\hline
\end{tabular}

Table 7: Fairness index.

\begin{tabular}{|c|c|}
\hline Number of MSs & Fairness \\
\hline \hline 5 & 0.998 \\
\hline 10 & 0.998 \\
\hline 15 & 0.995 \\
\hline 20 & 0.997 \\
\hline 25 & 0.997 \\
\hline 30 & 0.995 \\
\hline 35 & 0.997 \\
\hline 40 & 0.996 \\
\hline 45 & 0.997 \\
\hline 50 & 0.997 \\
\hline
\end{tabular}

the adjusted RED algorithm is used to measure the average queue length of each queue and monitor each connection states. As the average queue length exceeds the defined threshold, it represents that the connection is congested. The RS uses the congestion-aware extended subheader to inform its upstream MR-BS or RS of the connection ID and states. The upstream station uses the pause counter to stop the transmission of congested connection according to the reported state. Therefore, the saved bandwidth can be allocated to the normal connections to improve the network performance. We have modified the NIST WiMAX module to support IEEE $802.16 \mathrm{j}$ networks. Simulation results show that the throughput and dropping ratio are increased and reduced, respectively. Our future work is to investigate and devise the optimal pause counter by using the mathematical analysis.

\section{REFERENCES}

[1] Air interface for fixed broadband wireless access systems. IEEE 802.16-2004 Standard, October 2004.

[2] Air interface for fixed and mobile broadband wireless access systems amendment 2: Physical and medium access control layers for combined fixed and mobile operation in licensed bands and corrigendum 1. IEEE 802.16e-2005 Standard, February 2006.

[3] Air interface for fixed and mobile broadband wireless access systems multi-hop relay specification. IEEE 802.16j Draft Version 3, February 2008.

[4] S. Arun and P. Anand. Service criticality based scheduling for ieee 802.16 wirelessman. In IEEE Wireless Broadband and Ultra Wideband Communications, pages 12-16, Auguest 2007.

[5] D. Christakos, D. Cypher, N. Golmie, D. Griffith, K. Sriram, and et al. An IEEE 802.16 model for NS-2.
Seamless and Secure Mobility Project at National Institute of Standards and Technology in USA, April 2007.

[6] C. Cicconetti, A. Erta, L. Lenzini, and E. Mingozzi. Performance evaluation of the ieee 802.16 mac for qos support. IEEE Transactions on Mobile Computing, 6(1):26-38, January 2007.

[7] C. Cicconetti, L. Lenzini, E. Mingozzi, and C. Eklund. Quality of service support in ieee 802.16 networks. IEEE Network, 20(2):50-55, April 2006.

[8] S. Floyd and V. Jacobson. Random early detection gateways for congestion avoidance. IEEE/ACM Transactions on Networking, 1(4):397-413, August 1993.

[9] I. K. Fu, W. H. Sheen, C. L. Hsiao, and C. C. Tseng. System performance of relay-based cellular systems in manhattan-like scenario. IEEE C80216mmr-05/041, November 2005.

[10] I. K. Fu, W. H. Sheen, C. L. Hsiao, and C. C. Tseng. Throughput improvement with relay-augmented cellular architecture. IEEE C80216mmr-05/008, September 2005.

[11] I. K. Fu, W. H. Sheen, C. L. Hsiao, and C. C. Tseng. Reverse link performance of relay-based cellular systems in manhattan-like scenario. IEEE C80216mmr-06/004, January 2006.

[12] R. Jain, A. Durresi, and G. Babic. Throughput fairness index: An explanation. An Explanation, ATM Forum/99-0045, February 1999.

[13] M. Mehrjoo, X. Shen, and N. K. A joint channel and queue-aware scheduling for ieee 802.16 wireless metropolitan area networks. In IEEE Wireless Communications and Networking Conference, pages 1549-1553, March 2007.

[14] F. Ohrtman. WiMAX Handbook. McGraw-Hill, May 2005.

[15] A. Sayenko, O. Alanen, and T. Hamalainen. Scheduling solution for the ieee 802.16 base station. International Journal of Computer and Telecommunications Networking, 52(1):96-115, January 2008.

[16] A. Sayenko, O. Alanen, J. Karhula, and T. Hamalainen. Ensuring the qos requirements in 802.16 scheduling. In ACM International Workshop on Modeling Analysis and Simulation of Wireless and Mobile Systems, pages 108-117, October 2006.

[17] M. Shreedhar and G. Varghese. Efficient fair queuing using deficit round robin. IEEE Transactions on Networking, 4(3):375-385, June 1996.

[18] J. Sydir and K. Johnsson. Proposed text changes for downlink flow control. IEEE C802.16j-07/440r5, November 2007.

[19] K. Wongthavarawat and A. Ganz. Packet scheduling for qos support in ieee 802.16 broadband wireless access systems. International Journal of Communication Systems, 16(1):81-96, February 2003. 